\title{
The Four-Dimensional Fluid Space Research Program
}

\author{
Kennard Callender
}

\begin{abstract}
Finding a single theory that correctly answers the fundamental questions of nature and explains all physical phenomena is the major unsolved problem of theoretical physics. The Four-Dimensional Fluid Space Research Program is a plan that attempts to develop such a theory based on the hypothesis that physical space is a four-dimensional incompressible inviscid fluid. This article describes the program, the philosophy it follows, its fundamental postulates, and its objectives.
\end{abstract}

\section{Contents}

I Introduction i Problem Statement . . . . . . . . 1

ii Previous Research. . . . . . . . . 1

\begin{tabular}{|c|c|c|}
\hline \multicolumn{2}{|c|}{ II $\quad$ Research Program } & \\
\hline i & Research Philosophy & \\
\hline ii & Postulates & \\
\hline iii & Objectives & \\
\hline
\end{tabular}

III Conclusion

\section{INTRODUCTION}

$\mathrm{T}$ HE ultimate goal of theoretical physics is to formulate a single theory that fully explains all physical phenomena. This hypothetical theory is known as the theory of everything. Without it, true and complete understanding of nature is not possible. The focus on developing such a theory has been to create a mathematical framework that unifies quantum mechanics and general relativity. The problem of unification, however, remains unsolved. Therefore, it is reasonable to explore new approaches.

\section{i. Problem Statement}

The purpose of this paper is to describe the Four-Dimensional Fluid Space Research Program. This program is a plan that attempts to formulate a theory able to explain and predict all physical phenomena. The program requires the theory to be realistic, deterministic, and local. It should also be based on a few fundamental postulates that describe the nature of time, physical space, and elementary particles. The main hypothesis of the research is that physical space is a four-dimensional incompressible inviscid fluid.

\section{ii. Previous Research}

Almost every observed physical phenomena can be described either by quantum mechanics or general relativity. A theory of everything would need to make the same experimentally confirmed predictions those two theories have made. The main approach to develop a theory of everything has been to combine quantum mechanics and general relativity into a single mathematical framework. However, this objective has not been achieved yet.

The approach we propose here is to derive the equations of quantum mechanics and general relativity from a set of postulates that describe the nature of time, physical space, and elementary particles. These postulates are 
based on the hypothesis that physical space is a four-dimensional incompressible inviscid fluid. This hypothesis has been chosen as the foundation of our research because of its simplicity and explanatory power. Additionally, scientific literature confirms the potential it has.

In 1914, Gunnar Nordström developed a theory of gravity that assumed physical space was four-dimensional. In doing so, he ended up formulating the first unification between gravity and electromagnetism [1]. A few years later, Theodor Kaluza unified Einstein's general relativity with Maxwell's electromagnetism in a five-dimensional manifold (four dimensions of space and one of time) [2]. These unifications provide sufficient incentive to further explore the hypothesis that physical space is four-dimensional.

In 1926, Erwin Madelung derived Schrödinger's equation from the hydrodynamic equations for irrotational inviscid flows [3]. Even though Madelung did not identify physical space as the fluid in his interpretation, his derivation shows there is a connection between Schrödinger's equation and irrotational inviscid flows. This connection supports the idea that the mathematical formalism of quantum mechanics is actually describing physical space as an incompressible inviscid fluid undergoing irrotational flow.

\section{Research Program}

The Four-Dimensional Fluid Space Research Program is a plan that aims to develop a theory capable of answering the fundamental questions of nature and of explaining all physical phenomena. The plan consists of implementing a set of philosophical measures to guide the research process and to achieve the desired goal. After studying the mathematical structures of quantum mechanics and general relativity, the hypothesis that physical space is a four-dimensional incompressible inviscid fluid was chosen as the foundational principle of the research. We proceed to describe the program's research philosophy, its fundamental postulates, and its objectives.

\section{i. Research Philosophy}

To search for the theory of everything is to search for the truth. Thus, it makes sense to take a philosophical approach in formulating such a theory. In this section, we describe the philosophy our research program is based on.

Our research program starts by discarding all the physical objects and the philosophical principles proposed by modern physics. We assume only time, physical space, protons, and electrons exist. Time and physical space are considered to be fundamentally different. Our program also assumes the physical world is realistic, deterministic, and local. The mathematical formalism of non-relativistic quantum mechanics is taken to be accurate, but incomplete. The Lorentz transformations are also considered to be accurate, but misinterpreted.

The existence of protons and electrons is accepted because they are the only two types of massive particles that have been directly detected and that do not decay. Because of this, we refer to them as elementary particles in our research program. The philosophical worldview of our program requires the theory we develop to be realistic, deterministic, and local. Based on these assumptions and requirements, we formulated fundamental postulates about the nature of time, physical space, and elementary particles.

\section{ii. Postulates}

The Four-Dimensional Fluid Space Research Program is based on the following postulates:

- Time is absolute.

- Physical space is four-dimensional.

- Physical space is an incompressible inviscid fluid.

- The fluid flow of physical space is irrotational.

- Elementary particles always have a definite position and their movement is completely deterministic.

- Elementary particles are directly influenced only by their immediate surroundings. 
- Elementary particles move along the streamlines of the fluid flow of physical space at a constant speed.

- Based on their perception at the macroscopic level, observers assume physical space is three-dimensional, and this assumption has unexpected effects on the measurements they perform.

Additional postulates about the nature of elementary particles need to be added. That will be done, however, after achieving the preliminary objectives.

\section{iii. Objectives}

The ultimate goal of our research program is to formulate the theory of everything. However, in order to know if progress is being made, we have set three preliminary objectives. They consist of using the postulates from section 2.2 to (1) interpret the mathematical formalism of quantum mechanics, (2) derive Schrödinger's equation, and (3) derive the equations of special relativity.

The interpretation of quantum mechanics must be realistic, deterministic, and local. This means we must formulate a local hiddenvariable theory and address why Bell's theorem does not apply to it. If we achieve these three objectives, we can be confident that our postulates are valid. After that, we can add postulates about the nature of elementary particles to our research program.

\section{Conclusion}

In this paper we described the FourDimensional Fluid Space Research Program. The ultimate goal of the program is to formulate a theory that explains and predicts all physical phenomena. The main hypothesis of the research is that physical space is a fourdimensional incompressible inviscid fluid.

The research program sets some philosophical principles and fundamental assumptions that must be followed to develop the theory of everything. Based on these principles, we have formulated postulates that describe the nature of time, physical space, and elementary particles.

The program's worldview requires our formulation of the theory to be realistic, deterministic, and local. Preliminary objectives have been set to evaluate if progress is being made. We expect to publish the results obtained from these objectives in the near future.

\section{REFERENCES}

[1] Nordström, Gunnar. Uber die Moglichkeit, das Elektromagnetische Feld und das Gravitationsfeld $\mathrm{Zu}$ Vereinigen. (German) [On the Possibility of Unifying the Electromagnetic and the Gravitational Fields]. Phys. Z. 1914; 15: 504-506

[2] Kaluza, Theodor. Zum Unitätsproblem der Physik. (German) [On the Unification Problem in Physics]. Sitzungsberichte der Königlich Preußischen Akademie der Wissenschaften (Berlin). 1921; 1921: 966-972

[3] Madelung, Erwin. Quantentheorie in Hydrodynamischer Form. (German) [Quantum Theory in Hydrodynamical Form]. Zeitschrift für Physik. 1927; 40: 322-326 